\title{
An Improved Back-Propagation Neural Network for the Prediction of College Students' English Performance
}

\author{
https://doi.org/10.3991/ijet.v14i16.11187 \\ Wei Liu \\ Shandong University of Arts, Jinan, China \\ vivianliu516@163.com
}

\begin{abstract}
The global economic boom has greatly boosted the need for communication between different cultures and difference countries. The effective communication requires good command of foreign languages, especially English. This paper highlights the necessity to predict the English performance of college students, and sums up the types and features of neural network (NN) models. On this basis, the backpropagation (BP) NN was selected to predict the English performance of college students. The Spearman's R correlation test was conducted to analyze how the English performance is affected by the following factors: the score in National College Entrance Examination (NCEE), gender, age and learning attitude. Then, the improved BPNN was adopted to predict the English performance of college students. The results show that the NCEE score has the greatest impact on English performance, followed in descending order by learning attitude and gender, while age does not greatly affect English scores; the improved BPNN achieved a desirable effect in predicting the English performance of college students. The research findings shed new lights on college English teachers and learners.
\end{abstract}

Keywords—Neural network (NN), English performance, prediction, error

\section{Introduction}

As the progress of global economic integration accelerates, foreign languages are serving as a tool for people from different countries to communicate [1-5], and are playing an increasingly important role [6-10]. As an emerging and huge economic entity, China is a big country for foreign language learning, especially English learning, most students in China begin to learn English since the basic education stage, so China is also a big country for English learning.

Every year, tens of millions of students enter the higher education stage in China, and after which, they will enter the society. When facing the fierce competition in employment, English performance is an important indicator of employers. In this context, the research on English learning is of certain urgency, but currently there're few research results in this field. This paper uses Spearman's correlation test to find the influencing factors of college students' English scores, and adopts data mining to effectively predict college students' English scores, which can provide certain refer- 
ence for the targeted teaching of college English teachers, and it also plays a certain role in the improvement of students' achievements.

\section{Neural Network}

Artificial neural network (ANN) is an emerging artificial intelligence (AI) technology. Ever since the birth of AI, it has made remarkable progress [11-16]. The principle of ANN is to simulate the working modes of the human brain, and the calculation is performed by a large number of artificial "neurons" [17-18].

The "neurons" of ANN are divided into three types, one is the input layer neurons, the front end of neurons in this layer is not connected with other neurons, only the back end is connected with the lower layer neurons; one is the hidden layer neurons, which are connected with both upper layer and lower layer neurons, and are generally divided into single-layer and multiple-layer; the last one is the output layer neurons, which are opposite to the input layer neurons, the back end is not connected with other neurons, only the front end is connected with the upper layer neurons. ANN has a huge number of neurons, which represent the powerful information processing capability of ANN.

\subsection{Classification of ANN}

Commonly used ANNs are divided into the following types: feedforward neural networks, recurrent neural networks, and feedback neural networks.

Feedforward neural network: The feedforward neural network is a form of neural network that is relatively simple. The neurons are arranged hierarchically. The latter layer neurons are only connected to the previous layer neurons, and their input is determined by the output of the previous layer neurons. The feedforward neural network is characterized by unidirectional and forward information flow, and the output value is determined by the input value and the weight of the network. The typical structure of the feedforward neural network is shown in Figure 1.

In the figure, the yellow neurons in the first layer represent the input layer neurons; the green neurons in the second layer represent the hidden layer neurons; the yellow neurons in the third layer represent the output layer neurons.

Common feedforward neural networks include BP neural networks and RBF neural networks. For the nonlinear multilayer feedforward neural networks acting as universal approximators, BP neural network and RBF neural network have many common features. In some cases, they can replace each other. But there are also many differences between the two.

- BP neural network is a neural network with weight connection. RBF neural network has two connection modes: the connection between input layer and hidden layer is direct connection, and the connection between hidden layer and output layer is weight connection. 
- The transfer function of BP neural network generally chooses nonlinear function; while the transfer function of RBF generally selects the Gaussian function;

- The number of neurons in the BP neural network is stable, after the structure is determined, it will not change any more; while the number of neurons in the RBF can be adaptively adjusted in the training stage;

- The learning efficiency of BP neural network is fixed, and sometimes it takes a long time to complete the training; and the RBF neural network is a more efficient neural network;

- BP neural network is prone to be trapped in local minimum, while RBF neural network has the ability to achieve global optimization, and its accuracy is higher than BP neural network.

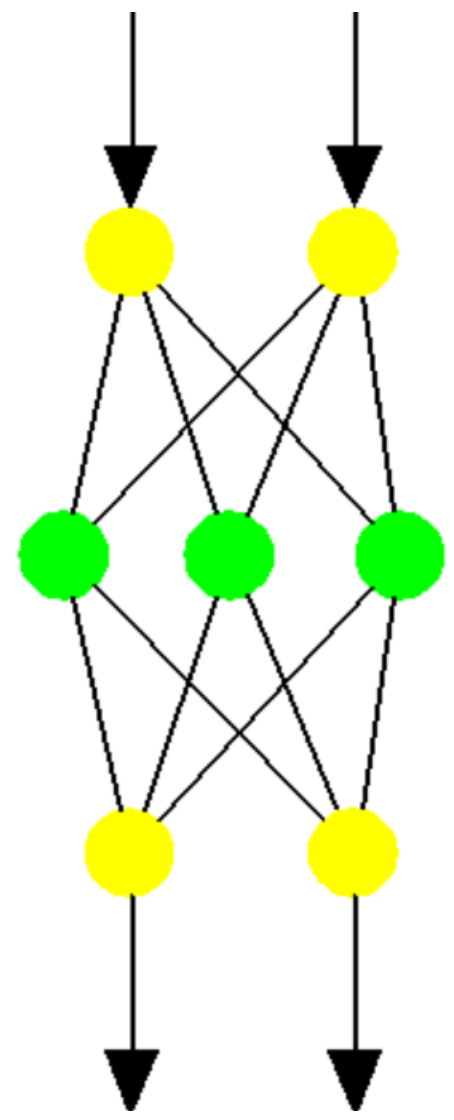

Fig. 1. Feedforward neural network

Recurrent neural network: The recurrent neural network is a tree-shaped neural network. One of its characteristics is that the topology is flexible and suitable for tasks involving structural relationships. One of the biggest differences between recurrent neural networks and feedforward neural networks is that the feedforward neural net- 
work only considers the current input, while the recurrent neural network considers not only the current input, but also the previously received information.

The information propagation method of recurrent neural network is shown in Figure 2.

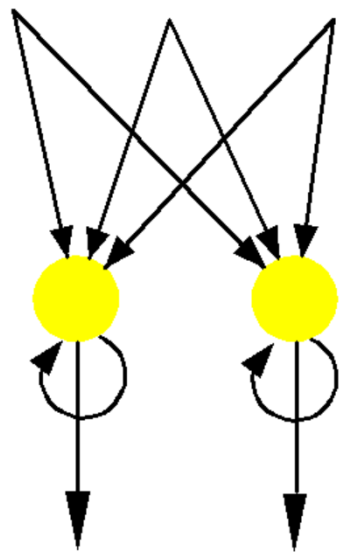

Fig. 2. Recurrent neural network

The recurrent neural networks consider both short-term memory and long-term memory, and have good performances in time series and text processing.

Feedback neural network: Compared with the feedforward neural network, the feedback neural network is a neural network with more powerful computing ability and stability. In the feedback neural network, there is no difference among the input neurons, hidden neurons, and output neurons, and the functions and status of all neurons are at the same level. Hopfield neural network is a relatively simple and common neural network in feedback neural networks. This neural network has associative memory and strong computing ability. Its structure is shown in Figure 3.

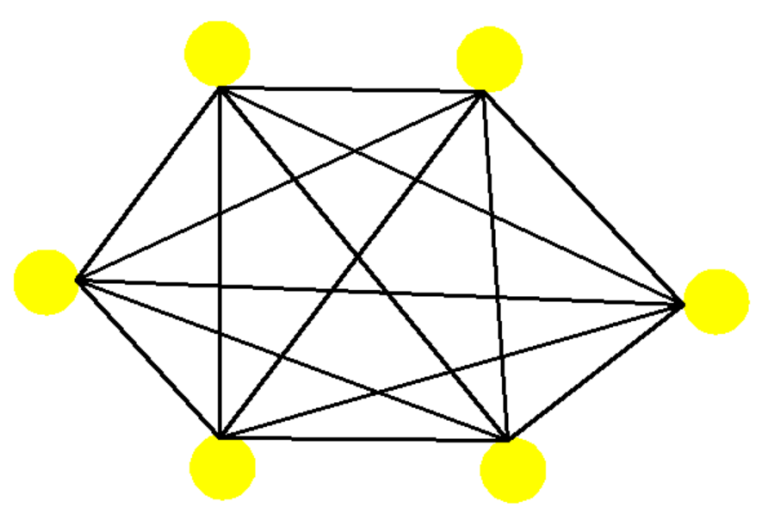

Fig. 3. Hopfield neural network 


\section{Analysis of Influencing Factors of College Students' English scores}

The English scores of college students are subject to many influencing factors. This paper took 101 freshmen's NCEE (National College Entrance Examination) score, gender, age and learning attitude as independent variables. The final exam scores by the end of the first semester of freshman year were studied as dependent variables, according to the correlation between independent variables and dependent variables, proper variables were selected.

\subsection{NCEE scores}

Before entering colleges, students already have many years of English learning experiences, and the learning foundation of previous learning stage will inevitably affect the learning outcomes of the college learning stage. Figures 4 and 5 show the distribution of NCEE scores and final exams results of the 101 college students respectively.

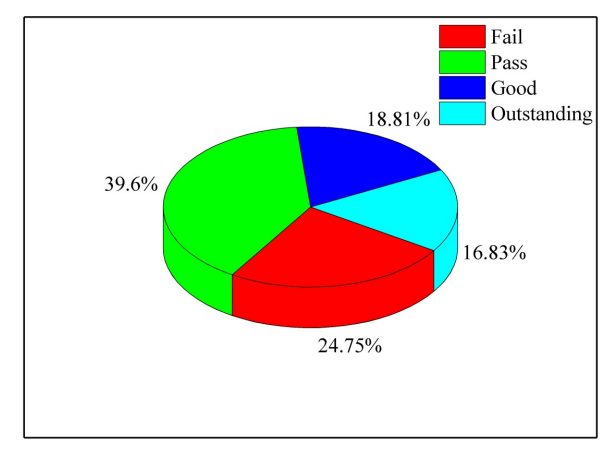

Fig. 4. Distribution of NCEE scores

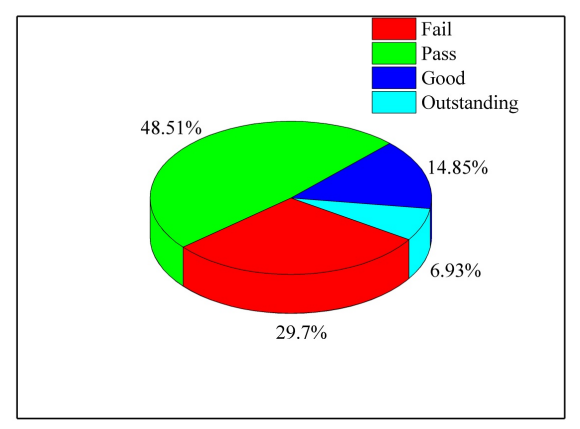

Fig. 5. Distribution of final exam results in the first semester 
In Figure 4 and Figure 5, the classification of NCCE scores and the classification of final exam results in the first semester are shown in Table 1.

Table 1. Test score classification

\begin{tabular}{|l|c|c|c|c|}
\hline \multicolumn{1}{|c|}{ Classification } & Fail & Pass & Good & Outstanding \\
\hline NCEE scores & $0 \sim 90$ & $90 \sim 120$ & $120 \sim 135$ & $135 \sim 150$ \\
\hline Final exam results for the first semester & $0 \sim 60$ & $60 \sim 80$ & $80 \sim 90$ & $90 \sim 100$ \\
\hline
\end{tabular}

As can be seen from Figure 4 and Figure 5, for the NECC scores of the 101 college students, the number of students with a score grade of "pass" was the most, followed by the number of students with a score grade of "fail", the number of students with a score grade of "good" was relatively fewer, while the number of students with a score grade of "outstanding" was the least. After one semester of study, the proportion of students of different score grades had changed to some extent. The proportion of students with a "pass" score grade had become larger, the proportion of students with a "fail" score grade had increased as well, the proportion of students with a "good" score grade had become smaller, and the proportion of students with an "outstanding" score grade had decreased as well. However, after these changes in the proportions, for the 101 college students, the number of students with a "pass" score grade was still the most, followed by the number of students with a "fail" score grade, the number of students with a "good" score grade was relatively fewer, and the number of students with an "outstanding" score grade was the least.

\subsection{Gender}

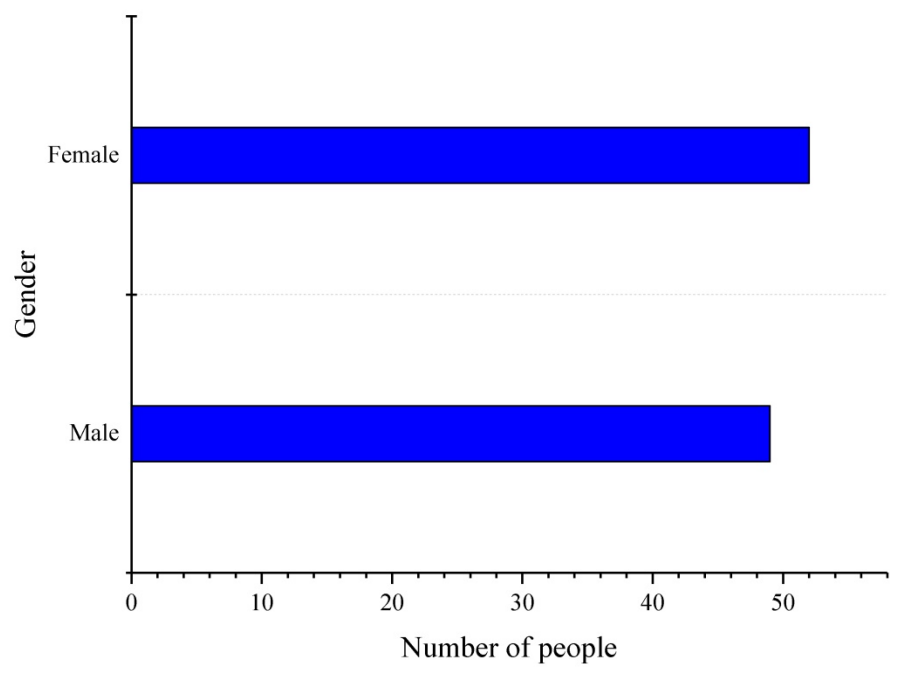

Fig. 6. Student gender distribution 
Students of different genders may have different learning habits. This paper took gender as an influencing factor of English performance. As can be seen from Figure 6, the ratios of male and female students in the 101 college students were roughly the same.

\subsection{Age}

Age has certain influence on students' learning ability, so this paper included age as an independent variable to be verified.

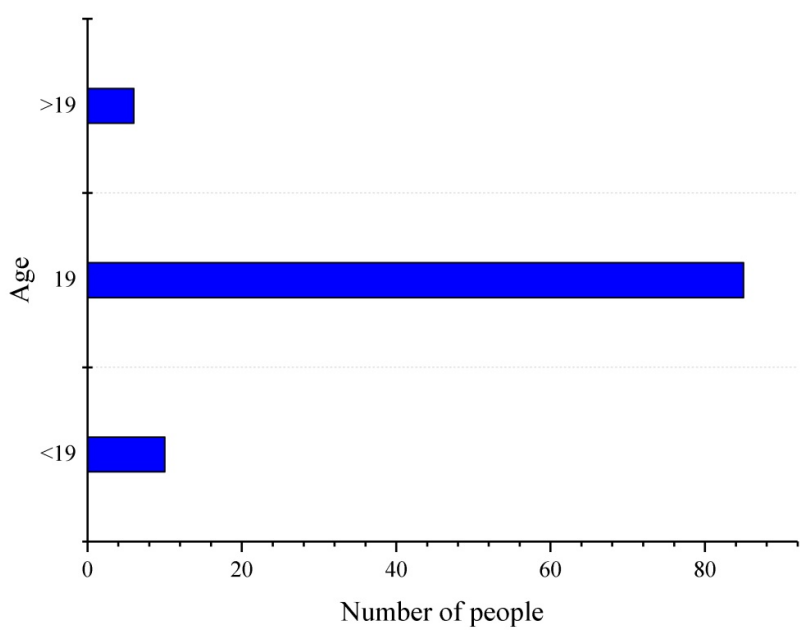

Fig. 7. Student age distribution

As shown in Figure 7, the age of most students was 19 years old, only a small number of students were older or younger than 19 , the number of students that were older than 19 was more than that were younger than 19.

\subsection{Learning attitude}

Learning attitude has a great impact on the learning performance. For the investigation of students' learning attitude, this paper adopted the subjective self-evaluation, and the score range was $0 \sim 100$ points, which was divided into four grades: $0-25$ points, $25-50$ points, $50-75$ points and $75-100$ points. According to statistics, the number of people who scored 50 to 75 points for their own learning attitude was the most, followed by the number of people who scored 25 to 50 points. The number of people who scored 0 to 25 points was fewer, and the number of people who scored 75 to 100 points was the least. In other words, most students gave themselves a middle- 
grade score for their learning attitude, while fewer gave a high-grade or a low-grade score.

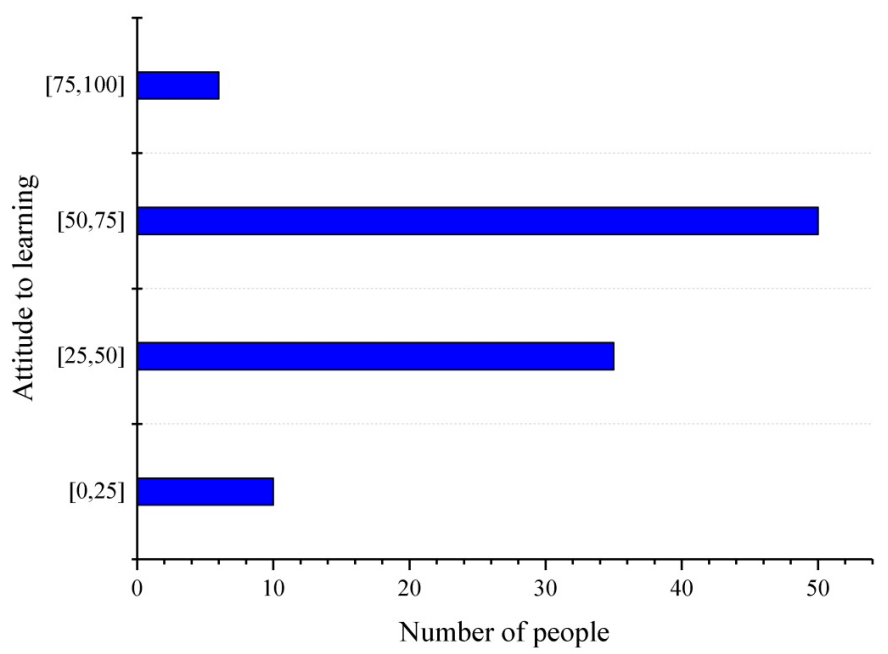

Fig. 8. Distribution of students' learning attitude

\subsection{Correlation test}

In order to verify whether the above factors can really affect the English scores of college students, this paper used the Spearman's correlation coefficient to test it. The results are shown in Table 2.

Table 2. Spearman's correlation test

\begin{tabular}{|l|c|c|}
\hline \multicolumn{1}{|c|}{ Factors } & Correlation coefficient & p \\
\hline NCEE score & 0.731 & $<0.01$ \\
\hline Gender & 0.386 & 0.04 \\
\hline Age & 0.189 & 0.12 \\
\hline Learning attitude & 0.471 & $<0.01$ \\
\hline
\end{tabular}

It can be seen from Table 2 that the NCEE score and learning attitude are the factors that have the greatest influence on the final exam results of the first semester. The correlation coefficients of the two were 0.731 and 0.471 , respectively, and the pvalues were all less than 0.01 , which means that both were positively correlated at a significant level of 0.01 , wherein the influence of NCEE score on the final exam results of the first semester was greater; second, gender factor was significantly positively correlated with the final exam results of the first semester at the level of 0.05 . In the correlation analysis, the gender of male was set to 0 , and the gender of female was set to 1, which means that female students' English performance was better; in addi- 
tion, the influence of age on the final exam results of the first semester was not significant, which may be because the age differences among students were not obvious.

\section{$4 \quad$ English Performance Prediction Model Based on Improved Neural Network}

\subsection{Improvement of BP neural network}

The BP neural network is simple in structure and is a commonly used artificial neural network, but it has some shortcomings as well. This paper chose BP neural network to realize the prediction of college students' English performance. However, before that, the BP neural network needs to be improved.

Determination of algorithm type: The obvious shortcomings of BP neural network are that its training speed is slow, and it is easy to fall into the local optimum. Different training algorithms may cause differences in the convergence speed of BP neural networks. After comparing the effects of different algorithms, it can be found that the Levenberg-Marquardt algorithm has the fastest convergence speed, and the smallest error in calculation. The only problem with this method is that it occupies a larger part of memory during calculation. Considering that the sample number of this study is relatively small, so the Levenberg-Marquardt algorithm was adopted for calculation.

Determination of the number of hidden layers and the number of nodes: In the training of BP neural network, the single-layered hidden layer was enough to approach any continuous function, and the three-layered hidden layer can correspond to any mapping relationship. The increase in the hidden layer number will lead to the increase in the training difficulty, and the improvement of prediction accuracy is limited. Therefore, the number of hidden layers selected in this paper was 1.

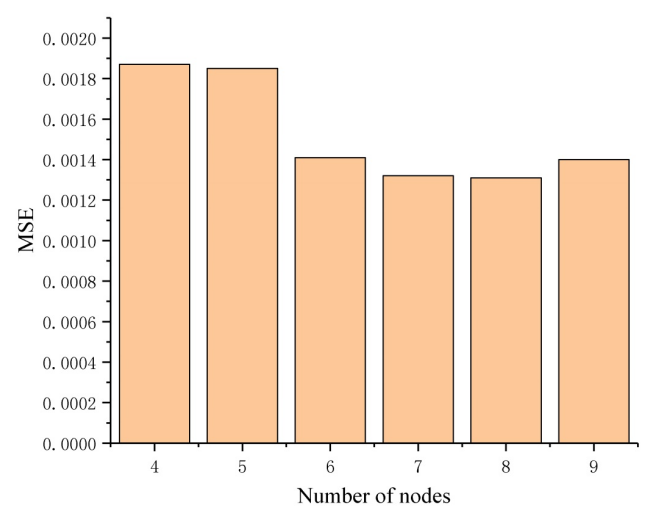

Fig. 9. Relationship between number of nodes and MSE 
The selection of the node number in the hidden layer has a great influence on the prediction accuracy of the model. Too-few node number would increase the training times of the model, and also affect the training precision; while too-many node number would prolong the training time of the model, even cause overfitting.

In this paper, the range of the selected hidden layer node number was 4-9. By comparing the prediction errors corresponding to different node number, the appropriate number of nodes was selected, and the results are shown in Figure 9. When the number of nodes gradually increased from 4 to 8 , the model prediction error showed a downward trend all the time, when the number of nodes increased from 5 to 6 , the variation range of the error was the largest. This paper selected 8 as the final hidden layer node number, in which case the prediction error was the smallest.

\subsection{Prediction effect of improved BP neural network}

This paper analyzed the prediction effect of the improved BP neural network from two aspects of model training times and relative errors. The results are shown in Figures 10 and 11 .

It can be seen from Figure 10 that after training for 20 times, the model error tended to be stable and close to 0 , and the model convergence effect was better. It can be seen from Figure 11 that although the model prediction accuracy varied for each student, for most students, the prediction effect was good, and the relative error was close to 0 .

In conclusion: the training times required by the model was not much; the prediction effect of the model on the college students' English performance was good; the prediction values can represent the distribution characteristics of the students' English scores; for some students, the relative error of the prediction value was relatively large, but for most students, the prediction error was close to 0 .

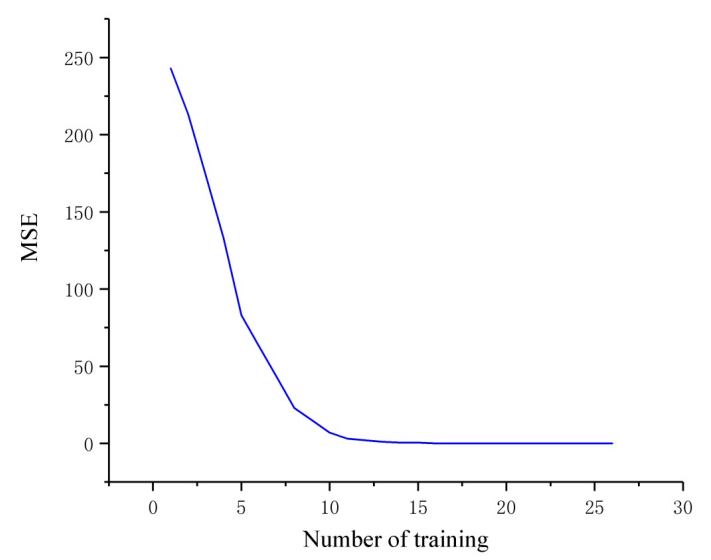

Fig. 10. Relationship between number of training and MSE 


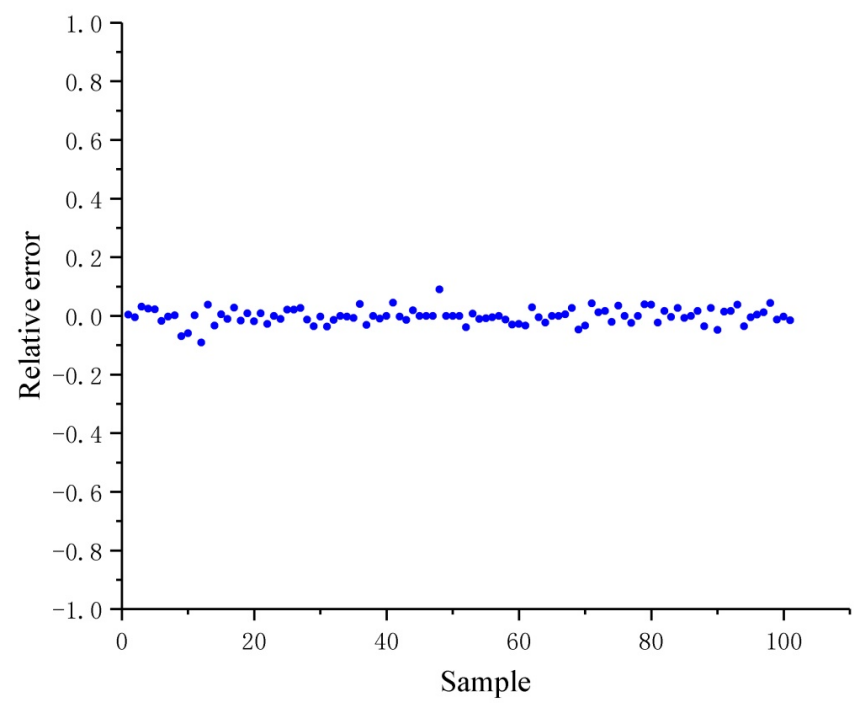

Fig. 11. Relative errors of the samples

\section{Conclusion}

This paper concluded the basic concepts in the neural network model, and summarized different types of neural networks. For the feedforward neural networks, recurrent neural networks and feedback neural networks, this paper summed up their computational ideas and basic features, and selected the BP neural network as the model in this paper;

This paper selected the NCEE score, gender, age and learning attitude as the independent variables, and the college students' first-term final English exam result as the dependent variable, through correlation test analysis, it's concluded that: the NCEE score has the greatest impact on the final exam results of the first semester, followed by the learning attitude and the gender factor, while age has little effect on English scores;

Through the improved BP neural network, the college students' English scores by the end of the first semester were predicted, and the results showed that, for the improved BP neural network, after the training time reached 20, the relative error of the model prediction tended to be stable, and the relative error was smaller, which means that the model prediction accuracy was higher. 


\section{Acknowledgement}

The study was supported by "Science and Technology Project of China Railway Corporation, China (Grant No. 1341324011)".

\section{$7 \quad$ References}

[1] Sparks, R. L., Ganschow, L., Patton, J. (1995). Prediction of performance in first-year foreign language courses: connections between native and foreign language learning. Journal of Educational Psychology, 87(4): 638-655. https://doi.org/10.1037//0022-0663.87.4.638

[2] Sparks, R. L., Patton, J. (2013). Relationship of 11 skills and 12 aptitude to 12 anxiety on the foreign language classroom anxiety scale. Language Learning, 63(4): 870-895. https://doi.org/10.1111/lang.12025

[3] Sparks, R. L. (1995). Examining the linguistic coding differences hypothesis to explain individual differences in foreign language learning. Ann Dyslexia, 45(1): 187-214. https://doi.org/10.1007/bf02648218

[4] Takano, Y., Noda, A. (1993). A temporary decline of thinking ability during foreign language processing. Journal of Cross-Cultural Psychology, 24(4): 445-462. https://doi.org/10.1177/0022022193244005

[5] Bundgaardnielsen, R. L., Best, C. T., Tyler, M. D. (2011). Vocabulary size is associated with second-language vowel perception performance in adult learners. Studies in Second Language Acquisition, 33(3): 433-461. https://doi.org/10.1017/s0272263111000040

[6] Felder, R. M., Henriques, E. R. (2010). Learning and teaching styles in foreign and second language education. Foreign Language Annals, 28(1): 21-31. https://doi.org/10.1111 j.1944-9720.1995.tb00767.x

[7] Stryker, S. B. E., Leaver, B. L. E. (1997). Content-based instruction in foreign language education: models and methods. Hispania, 82(1): 95. https://doi.org/10.2307/346085

[8] Tulasiewicz, W. (2010). A review of "from foreign language education to education for intercultural citizenship: essays and reflections". Language Awareness, 19(3): 227-229. https://doi.org/10.1080/09658416.2010.519171

[9] Distler, O., Huber, L. C., Ha, H. R., Simmen, B., Kalden, J. R., Pisetsky, D. S. (2007). On national foreign language education policy:a new strategic perspective. Foreign Language World, 56(11): 3564-3574. http://dx.doi. org/10.1002/art.22980

[10] Byram, M. (1988). Foreign language education and cultural studies. Language Culture \& Curriculum, 1(1): 15-31. https://doi.org/10.1080/07908318809525025

[11] Chandra, P. (2003). Sigmoidal function classes for feedforward artificial neural networks. Neural Processing Letters, 18(3): 205-215. https://doi.org/10.1023/b:nepl.0000011137. $\underline{04221.96}$

[12] Huang, G. B., Zhu, Q. Y., Siew, C. K. (2006). Extreme learning machine: theory and applications. Neurocomputing, 70(1-3): 489-501. https://doi.org/10.1016/j.neucom.2005. $\underline{12.126}$

[13] Hinton, G. E., Srivastava, N., Krizhevsky, A., Sutskever, I., Salakhutdinov, R. R. (2012). Improving neural networks by preventing co-adaptation of feature detectors. Computer Science, 3(4): 212-223. http://dx.doi.org/10.9774/GLEAF.978-1-909493-38-4 2

[14] Sánchez-Escalona. A.A., Góngora-Leyva, E. (2018). Artificial neural network modeling of hydrogen sulphide gas coolers ensuring extrapolation capability, Mathematical Modelling of Engineering Problems, 5(4), 348-356. https://doi.org/10.18280/mmep.050411 
[15] Mostefa, T., Tarak, B., Hachemi, G. (2018). An automatic diagnosis method for an open switch fault in unified power quality conditioner based on artificial neural network, Traitement du Signal, 35(1), 7-21. https://doi.org/10.3166/ts.35.7-21

[16] Choubey, H., Pandey, A. (2018). Classification of healthy, inter-ictal and seizure signal using various classification techniques, Traitement $\mathrm{du}$ Signal, 35(1), 75-84. https://doi.org/10.3166/ts.35.75-84

[17] Yao, X. (1999). Evolving artificial neural networks. Proceedings of the IEEE, 87(9): 14231447. http://dx.doi. org/10.1109/5.784219

[18] Hassoun, M. H. (2002). Fundamentals of artificial neural networks. Proceedings of the IEEE, 84(6): 906. http://dx.doi. org/10.1109/JPROC.1996.503146

\section{Author}

Wei Liu, female, was born on March 10, 1982, with a Master's degree, major in English Language and Literature. She is currently working as an English teacher at Shandong University of Arts in Jinan, China. She has been teaching English courses for 11 years, including College English course and Listening \&Speaking course. She has published one book and several papers on college English teaching. She has been excellent in annual assessment for several times.

Article submitted 2019-05-23. Resubmitted 2019-07-07. Final acceptance 2019-07-12. Final version published as submitted by the authors. 